\title{
Découverte fortuite d'une lésion mandibulaire, un cas de kyste anévrismal
}

\author{
C. Gossiome (Paris), F. Rufino (Paris), G. Herve (Paris), M. Benassarou (Paris), P. Goudot \\ (Paris), V. Descroix (Paris), G. Lescaille (Paris)
}

Introduction : Les kystes et tumeurs des maxillaires représentent une multitude d'entités pour lesquelles le diagnostic est parfois difficile. L'examen anatomopathologique permet dans la majorité des cas de donner un diagnostic de certitude mais il est parfois nécessaire de confronter ces résultats à l'imagerie voir de réaliser des marquages d'immunohistochimie pour mieux caractériser la lésion. Le diagnostic différentiel apparaît comme primordial devant certaines entités dont l'évolution guide une prise en charge très différente. Cas clinique : Il s'agissait d'une jeune patiente de 25 ans sans antécédent médical, adressée par son chirurgien-dentiste à la suite de la découverte fortuite d'une lésion osseuse au niveau de l'angle mandibulaire droit. Une exploration radiographique de type CBCT ainsi qu'une biopsie osseuse ont été réalisées. La confrontation de l'imagerie et du diagnostic histologique ne permettait pas de dissocier deux diagnostics possibles, la lésion pouvant correspondre à une tumeur à cellules géantes (TCG) ou à un kyste osseux anévrismal (KOA). Devant cette incertitude, un marquage par immunohistochimie de la protéine p63 ainsi qu'une imagerie par résonance magnétique ont été demandés. Un marquage négatif à la p63 ainsi que l'analyse de l'ensemble des images radiologiques nous a permis de poser le diagnostic de kyste anévrismal. Devant le caractère asymptomatique de la lésion et de l'absence d'évolution à 9 mois, une attitude de surveillance a été décidée. Discussion : Les deux entités que sont le KOA et la TCG sont des lésions dont le diagnostic différentiel est complexe mais impératif du fait de leurs évolutions très différentes. Le KOA correspond à une dystrophie osseuse bénigne qui forme une lésion cavitaire constituée d'un réseau riche en fibroblastes et cellules géantes plurinucléés parfois bordées par un endothélium. Cette lésion est bénigne d'évolution lente. Le traitement consiste le plus souvent en un curetage de la cavité kystique lorsque la lésion entraîne déformation et/ou symptomatologie. La TCG présente de nombreuses caractéristiques histologiques en commun avec le kyste osseux anévrismal, avec un nombre de cellules géantes plus important. Toutefois la TCG présente un risque de récidive de l'ordre de $50 \%$ ainsi qu'un risque de transformation maligne (sarcome) de 10 à $20 \%$. Son évolution peut également être rapide. Le traitement doit être radical et consiste en une exérèse chirurgicale avec marges. Il a été montré par plusieurs auteurs que le marquage de p63, une protéine nucléaire de la famille du gène suppresseur p53, retrouvée dans différents tissus permettait de distinguer ces deux entités dans plusieurs localisations. Conclusion : Le marquage de la protéine p63 peut apparaît donc très utile dans le diagnostic différentiel de ces lésions dont les pronostics sont très différents lorsque l'imagerie et le marquage en HES ne sont pas suffisants.

Références : Shooshtarizadeh T. P63 expression as a biomarker discriminating giant cell tumor of bone from other giant cell-rich bone lesions. Pathol Res Pract. 2016 Oct;212(10):876-879 